\title{
SEGMENTATION-FREE STATISTICAL IMAGE RECONSTRUCTION FOR POLYENERGETIC X-RAY COMPUTED TOMOGRAPHY
}

\author{
Idris A. Elbakri and Jeffrey A. Fessler, Senior Member, IEEE \\ Electrical Engineering and Computer Science Department \\ University of Michigan \\ Ann Arbor, MI. \\ Email: ielbakri@umich.edu
}

\begin{abstract}
This paper describes a statistical iterative reconstruction method for X-ray CT based on a physical model that accounts for the polyenergetic $\mathrm{X}$-ray source spectrum and the measurement nonlinearities caused by energy-dependent attenuation. The algorithm accommodates mixtures of tissues with known mass attenuation coefficients but unknown densities. We formulate a penalized-likelihood approach for this polyenergetic model based on Poisson statistics.
\end{abstract}

\section{INTRODUCTION}

There has been recently a surge of interest in applying statistical reconstruction techniques to $\mathrm{X}$-ray computed tomography $[1,2]$. Statistical techniques are better suited than the classical filtered backprojection for cone-beam and helical scanning geometries. They also offer better bias-variance performance, lower dose, and can incorporate accurate models of data acquisition and the physics involved $[3,4,5]$. Their main drawback (relative to FBP) is longer computation times.

Most prior statistical CT reconstruction algorithms assume monoenergetic X-ray beams, which is incompatible with clinical systems. Using a monoenergetic algorithm for polyenergetic data results in severe beam hardening artifacts $[6,7]$. In this paper we present and demonstrate the effectiveness of a statistical reconstruction algorithm for X-ray CT that is based on a polyenergetic model.

The classical beam hardening correction method described by Joseph and Spital (henceforth referred to as JS) [8], corrects for distortions caused by soft-tissue and high $\mathrm{Z}$ materials (bone). It requires knowledge of the X-ray spectrum and a pre-segmented initial image. It involves linearization and a final reconstruction.

In earlier work [7, 6], we proposed a statistical algorithm for beam hardening correction that required knowledge of the X-ray spectrum and, like JS, required a pre-

This work was supported in part by NIH grant R01CA/HL60711-06. segmented initial image (usually obtainable from a good FBP image). The approach assumed that the different tissue types in the object did not overlap.

Recently, De Man et al. suggested an alternative statistical approach [9] that models the object attenuation as a linear combination of the Compton scatter and photoelectric effect of two base substances. Prior knowledge of the $\mathrm{X}$-ray spectrum is required, but a pre-segmented image is not. The algorithm can reconstruct mixed pixels but is not guaranteed to be monotonic.

In this paper, we generalize our earlier model by allowing pixels to contain tissue mixtures. We introduce an improved object model that eliminates the need for presegmenting bone and soft tissue. The algorithm iteratively minimizes surrogate functions to the Poisson likelihood. Ordered subsets accelerate the algorithm. The algorithm can accommodate scatter and one version thereof is monotonic.

\section{POLYENERGETIC X-RAY CT}

The linear attenuation coefficient $\mu(x, y, z, \mathcal{E})$ characterizes the overall attenuation property of an object. It depends on the spatial coordinates $(x, y, z)$ and the beam energy $\mathcal{E}$, and has units of inverse distance. For a ray $L_{i}$ of infinitesimal width, the projection measurement $Y_{i}$ recorded by the $i$ th detector would ideally be

$$
Y_{i}=\int I_{i}(\mathcal{E}) e^{-\int_{L_{\boldsymbol{i}}} \mu(x, y, z, \mathcal{E}) d l} d \mathcal{E} .
$$

$I_{i}(\mathcal{E})$ incorporates the energy dependence of the incident ray and the detector sensitivity. The goal of CT reconstruction is to recover the attenuation coefficient from measured projection data $\left\{Y_{i}\right\}_{i=1}^{N}$, where $N$ is the number of rays. In reality, the measurements suffer from scatter and noise.

If the reconstruction algorithm disregards the energy dependence of $\mu$ in (1), artifacts such as dark streaks between bones and cupping in soft tissue appear $[10,8]$.

In this section we summarize an object model for polyenergetic X-ray attenuation and a statistical model for the data. 


\subsection{Polyenergetic Object Model}

We assume that each pixel in the object may contain a mixture of up to $K$ materials. We model the attenuation coefficient of the $j$ th pixel as the sum over $K$ materials of the product of the (known) energy-dependent mass attenuation coefficient $m_{k}(\mathcal{E})\left(\mathrm{cm}^{2} / \mathrm{g}\right)$, the (unknown) energyindependent density $\rho_{j}\left(\mathrm{~g} / \mathrm{cm}^{3}\right)[11,8,12]$ and the material fraction at the pixel $f_{j}^{k}$. Expressed mathematically in the discrete domain,

$$
\begin{aligned}
\mu(x, y ; \mathcal{E}) & =\sum_{j=1}^{p} \mu_{j}(\mathcal{E}) b_{j}(x, y) \\
& =\sum_{j=1}^{p} \sum_{k=1}^{K} m_{k}(\mathcal{E}) \rho_{j} f_{j}^{k}\left(\rho_{j}\right) b_{j}(x, y)
\end{aligned}
$$

where $b_{j}(x, y)$ is an appropriate spatial basis function, e.g., voxels. We require that $f_{j}^{k}\left(\rho_{j}\right) \geq 0$, and $\sum_{k} f_{j}^{k}=1$.

We denote the system matrix by $\mathbf{A}=\left\{a_{i j}\right\}$ where $a_{i j}=$ $\int_{L_{i}} b_{j}(x, y) d l$. The line integral of the attenuation coefficient is

$$
\int_{L_{i}} \mu(x, y ; \mathcal{E}) d l=\sum_{j=1}^{p} \sum_{k=1}^{K} m_{k}(\mathcal{E}) \rho_{j} f_{j}^{k}\left(\rho_{j}\right) a_{i j}
$$

By defining $s_{i}^{k}(\rho) \triangleq \sum_{j=1}^{p} a_{i j} f_{j}^{k}\left(\rho_{j}\right) \rho_{j}$ and $\underline{s}_{i}(\rho)=$ $\left(s_{i}^{1}, s_{i}^{2}, \ldots, s_{i}^{K}\right)$ we express the mean of the measured data along path $L_{i}$ as

$$
E\left[Y_{i}\right]=\int I_{i}(\mathcal{E}) e^{-\underline{m}^{\prime}(\mathcal{E}) \underline{s}_{i}(\rho)} d \mathcal{E}+r_{i} \triangleq \bar{Y}_{i}\left(\underline{s}_{i}(\rho)\right)+r_{i}
$$

where $r_{i}$ represents scatter and other background signal, and $\underline{m}^{\prime}(\mathcal{E})=\left[m_{1}(\mathcal{E}), \ldots, m_{K}(\mathcal{E})\right]$. We have expressed the measurements as a function of the vector $\underline{s}_{i}$ which has as its elements the line integrals of the $K$ different material densities. Given the $X$-ray spectrum, we tabulate the values of $\bar{Y}_{i}(\cdot)$ and its gradient $\nabla \bar{Y}_{i}(\cdot)$ over the range of arguments $\left(s^{1}, \ldots, s^{K}\right)$ that correspond to representative objects for later use in the iterative algorithm [7].

To keep the number of unknowns equal to $p$, we let the material fractions be functions of pixel density. We next explore the form of these functions $f_{j}^{k}\left(\rho_{j}\right)$ for $K=2$.

\subsection{Tissue Fraction Functions $\left\{f_{k}^{j}\left(\rho_{j}\right)\right\}$}

Fig. 1 illustrates the normalized mass attenuation coefficients of several human tissues in the diagnostic X-ray energy range [13]. Most soft tissue have spectral characteristics and densities that are very similar to those of water. We therefore use water and bone as our base materials. More base substances, such as Iodine, can be added as needed.

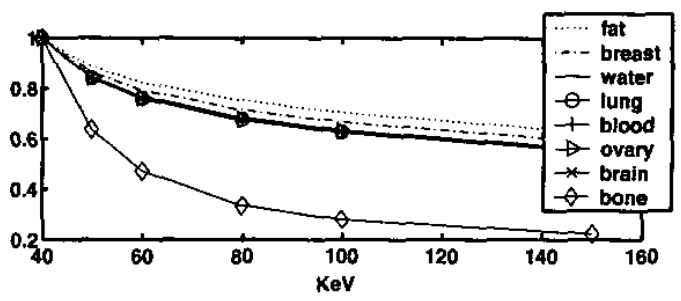

Fig. 1. Mass attenuation coefficient of human tissues normalized at $40 \mathrm{KeV}$

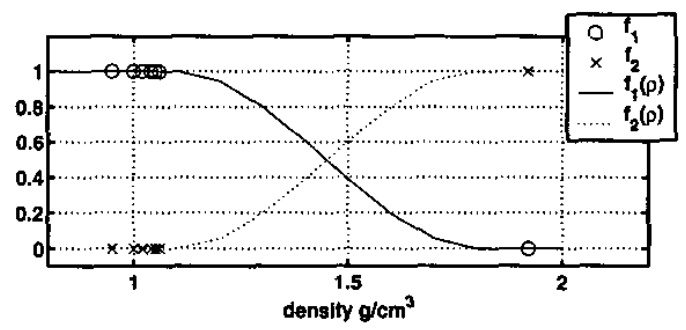

Fig. 2. Water and bone coefficient computed using constrained weighted least squares

We set $K=2$ in (2) and model the attenuation coefficient of tissue with the following approximation:

$\mu(x, y ; \mathcal{E}) \approx \sum_{j=1}^{p}\left(m_{1}(\mathcal{E}) f_{1}^{j}\left(\rho_{j}\right)+m_{2}(\mathcal{E}) f_{2}^{j}\left(\rho_{j}\right)\right) \rho_{j} b_{j}(x, y)$

where $m_{1}(\mathcal{E})$ and $m_{2}(\mathcal{E})$ are the mass attenuation coefficients of water and bone, respectively. The pixel tissue fraction functions $f_{1}^{j}$ and $f_{2}^{j}$ determine to what extent the tissue in pixel $j$ is spectrally water-like or bone-like respectively, depending on the tissue density.

To gain insight into possible forms of $f_{j}^{1}$ and $f_{j}^{2}$ we use weighted (by the beam spectrum) least squares to compute the coefficients $f_{1}$ and $f_{2}$ in

$$
m(\mathcal{E})=m_{1}(\mathcal{E}) f_{1}+m_{2}(\mathcal{E}) f_{2}
$$

for the substances shown in Fig. 1. The coefficients were constrained to lie in the interval $[0,1]$ and to satisfy $f_{1}+$ $f_{2}=1$. The results are plotted in Fig. 2 as a function of density.

One possibility that emerges from Fig. 2 would be to set $f_{1}(\rho)=1$ below a certain density threshold and 0 otherwise. This would segment all pixels into either bone or water, and does not permit mixtures, which is essentially our earlier approach $[6,7]$. 
Another approach would be to linearly interpolate by making the $f_{j}^{k}\left(\rho_{j}\right)$ functions piecewise linear. This effectively assumes that all substances lie on a straight line between the water (density $1.0 \mathrm{~g} / \mathrm{cm}^{3}$ ) and bone (density 1.92 $\mathrm{g} / \mathrm{cm}^{3}$ ) coefficients. This is similar to the approach of De Man et al., [9]. A disadvantage of this piecewise linear approach is that the derivatives of $f_{j}^{k}\left(\rho_{j}\right)$ would have complicating discontinuities.

To model mixed pixels, we propose using the functions plotted in Fig. 2 using the solid and dotted lines. These are third order polynomials functions of the tissue density that have continuous first and second derivatives, and that satisfy $f_{1}(\rho)+f_{2}(\rho)=1$. This choice essentially models tissues that have densities close to that of water and that are spectraily similar to water as 'dense water'. The model allows these tissues to have densities different from water, while assuming that they are spectrally 'similar' to water. The data if Fig. 2 shows that this model agrees with published values better than linear interpolation.

\subsection{Statistical Model and Iterative Algorithm}

We use a penalized-likelihood approach to reconstruct the data. For simplicity, we model the measurements as independently distributed Poisson random variables with mean given by (4), but the method easily generalizes [14]. The Poisson negative log-likelihood is:

$$
-L(\rho)=\sum_{i=1}^{N} h_{i}\left(\tilde{Y}_{i}\left(\underline{s}_{i}(\rho)\right)+r_{i}\right)
$$

where $h_{i}(t) \triangleq-Y_{i} \log t+t$. The reconstruction problem now is to find an estimate that minimizes $-L(\rho)$ given such physical constraints as nonnegativity. To derive an iterative algorithm that minimizes the cost function, we use optimization transfer techniques similar to those in $[7,5]$. The final update step takes the form:

$$
\rho^{\text {new }}=\rho^{\text {old }}-\mathbf{D}^{-1} \nabla^{\prime} L\left(\rho^{\text {old }}\right)
$$

where $\mathbf{D}$ is a diagonal matrix whose entries affect convergence. In practice, we include regularization because the problem is ill-conditioned and use ordered-subsets to accelerate the algorithm.

\section{PRELIMINARY RESULTS}

We simulated parallel-beam transmission Poisson data of the density phantom in Fig. 3a [15] with a spectrum that has mean $67.12 \mathrm{keV}$ and standard deviation $17.76 \mathrm{keV}$ [16]. The bones and soft tissue have densities $1.8-2.0 \mathrm{~g} / \mathrm{cm}^{3}$ and $0.2-1.0 \mathrm{~g} / \mathrm{cm}^{3}$ respectively. The FOV is $51 \mathrm{~cm}$ and the sinogram has 1200 radial bins and 1200 angular steps over $180^{\circ}$. We did not simulate background events. The blank scan value was $4.87 \times 10^{6} \mathrm{counts} /$ detector. The original phantom was $1024 \times 1024$ but we reconstructed the data on a $512 \times 512$ grid to generate the effect of mixed pixels. Uncorrected FBP, JS-corrected FBP and the iterative algorithm were applied to the data. The iterative algorithm ran for 10 iterations with 50 subsets and used edge-preserving shift-variant regularization [17].

Fig. 3 shows the results. The uncorrected FBP image (not shown) suffered from severe beam hardening artifacts. The statistical iterative reconstruction exhibits significantly better artifact reduction than the Joseph and Spital image because it inherently models the nonlinearity and reconstructs the data accordingly.

\section{SUMMARY}

We present and demonstrate the effectiveness of a polyenergetic statistical X-ray CT reconstruction algorithm. The algorithm outperforms the JS classical approach in the case of a soft tissue and bone phantom. In future work, we will apply the algorithm to read data and will further refine the physical model.

\section{REFERENCES}

[1] B. De Man, J. Nuyts, P. Dupont, G. Marchal, and P. Suetens, "Reduction of metal artifacts in X-ray computed tomography using a transmission maximum a posteriori algorithm," IEEE Transactions on Nuclear Science, vol. 47, no. 3, pp. 977-981, June 2000.

[2] A. H. Delaney and Y. Bresler, "Globally convergent edge-preserving regularized reconstruction: an application to limited-angle tomography," IEEE Transactions on Image Processing, vol. 7, no. 2, pp. 104-221, February 1998.

[3] J. A. Fessler, E. P. Ficaro, N. H. Clinthorne, and K. Lange, "Grouped-coordinate ascent algorithms for penalized likelihood transmission image reconstruction," IEEE Transactions on Medical lmaging, vol. 16, no. 2, pp. 166-175, April 1997.

[4] C. Kamphius and F. J. Beekman, "Accelerated iterative transmission CT reconstruction using an ordered subsets convex algorithm," IEEE Transactions on Medical lmaging, vol. 17, no. 6, pp. 1101-1105, December 1998.

[5] H. Erdoğan and J. A. Fessler, "Ordered subsets algorithms for transmission tomography," Physics in Medicine and Biology, vol. 44, pp. 2835-2851, 1999. 


\section{a. True Object}

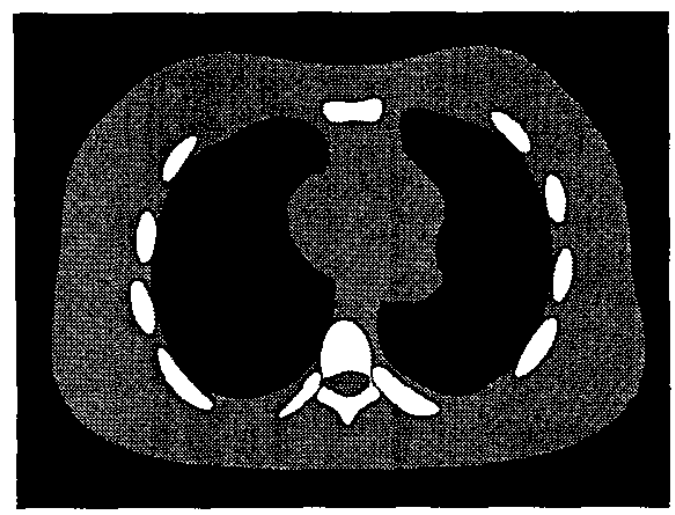

\section{b. JS-corrected FBP}

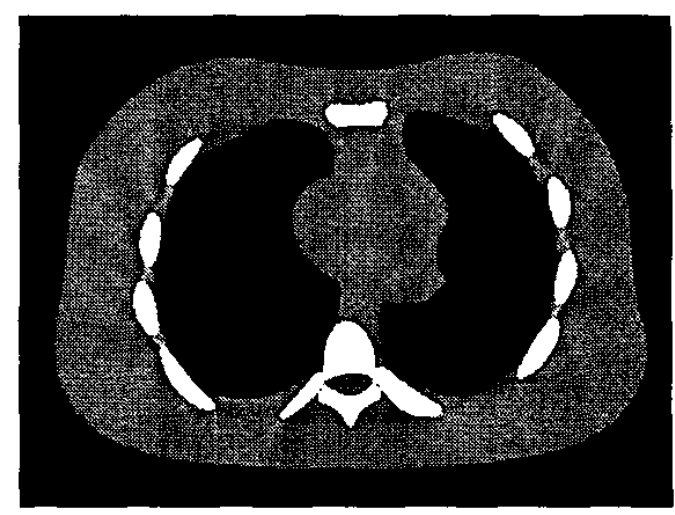

\section{c. Statistical Reconstruction}

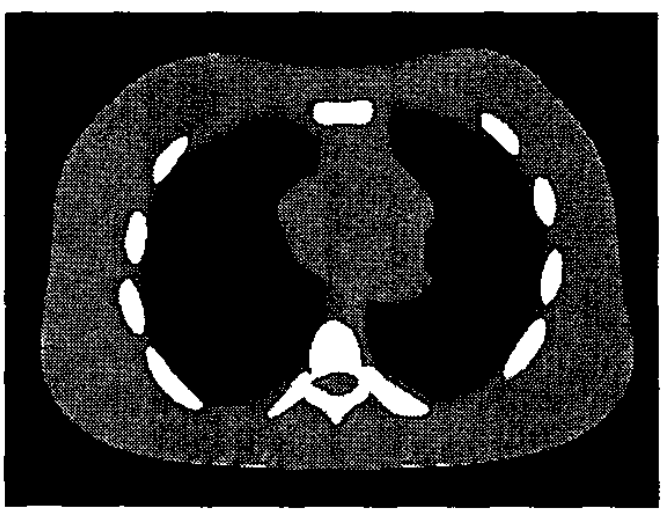

Fig. 3. Beam Hardening Correction Results. Window Range $=\left[\begin{array}{ll}.8 & 1.2\end{array}\right]$.
[6] I. El Bakri and J. A. Fessler, "Ordered subsets transmission reconstruction with beam hardening correction for x-ray CT," in Proc. SPIE 4322, Medical Imaging 2001: Image Proc., 2001, vol. 1, pp. 1-12.

[7] I Elbakri and J. A. Fessler, "Statistical image reconstruction for polyenergetic X-ray computed tomography," IEEE Tr. Med. Im., vol. ?, 2001, Submitted.

[8] P. M. Joseph and R. D. Spital, "A method for correcting bone induced artifacts in computed tomography scanners," Journal of Computer Assisted Tomography, vol. 2, pp. 100-108, January 1978.

[9] Bruno De Man, Johan Nuyts, Patrick Dupont, Guy Marchal, and Paul Suetens, "An iterative maximumlikelihood polychromatic algorithm for CT," IEEE Tr. Med. Im., vol. 20, no. 10, pp. 999-1008, Oct. 2001.

[10] R. A. Brooks and G. D. Chiro, "Beam hardening in Xray reconstruction tomography," Physics in Medicine and Biology, vol. 21, no. 3, pp. 390-398, 1976.

[11] R. E. Alvarez and A. Macovski, "Energy-selective reconstruction in X-ray computerized tomography," Physics in Medicine and Biology, vol. 21, no. 5, pp. 733-744, 1976.

[12] P. Sukovic and N. H. Clinthome, "Penalized weighted least-squares image reconstruction in single and dual energy X-ray computed tomography," IEEE Transcations on Medical Imaging, vol. 19, no. 11, pp. 107581, November 2000.

[13] J. H. Hubbell and S. M. Seltzer, "Tables of $\mathrm{x}$-ray mass attenuation coefficients and mass energy-absorption coefficients," http://physics.nist.gov/PhysRefData/XrayMassCoef/.

[14] Jeffrey A Fessler, Idris Elbakri, Predrag Sukovic, and Neal H Clinthorne, "Maximum-likelihood dualenergy tomographic image reconstruction," in spie, 2002, To appear. 4684-05.

[15] B. M. W.Tsui, J. A. Terry, and G. T. Gullberg, "Evaluation of cardiac cone-beam SPECT using observer performance experiments and roc analysis," Investigative Radiology, vol. 28, no. 12, 1993.

[16] P. Sukovic and N. H. Clinthorne, "Design of an experimental system for dual energy X-ray CT," in Proc. IEEE Nuc. Sci. Symp. Med. Im. Conf., 1999, vol. 2, pp. $1021-2$.

[17] J. A. Fessler and W. L. Rogers, "Spatial resolution properties of penalized-likelihood image reconstruction methods: Space-invariant tomographs," IEEE Tr. Im. Proc., vol. 5, no. 9, pp. 1346-58, Sept. 1996. 